\title{
EDUCATION SYSTEM FOR ELECTRONIC CIRCUIT CONSTRUCTION INVOLVING SOLDERING ON A CIRCUIT BOARD
}

\author{
Atsushi Takemura \\ Tokyo University of Agriculture and Technology \\ 2-24-16 Naka-cho, Koganei-shi, Tokyo 184-8588, Japan
}

\begin{abstract}
This paper proposes a novel education system for learning electronic circuit construction involving soldering on a universal circuit-board. This system enables a student to efficiently design the layout of circuit components on a circuit board and helps the student securely construct the circuit by soldering the circuit components in accordance with the designed layout. Web-based instructions are utilized for wiring and soldering the circuit components on the lower surface of the circuit-board based on automated recognition of circuit images during the circuit construction. The effectiveness of the proposed system was verified by 40 undergraduate students at Tokyo University of Agriculture and Technology. The comparison tests between two groups that comprised students that used or did not use the proposed system demonstrated the effectiveness of the system.
\end{abstract}

\section{KEYWORDS}

Web-Based Education, Circuit Construction, Soldering

\section{INTRODUCTION}

Knowledge of electronic circuit construction and practical applications, such as signal processing, machine control, and robotics, are of critical importance for a modern technology education. Recently, several educational support systems have been developed to improve students' understanding of electronic circuits and their ability for circuit construction (Reisslein, 2013; Rodriguez-Sanchez, 2016; Garcia-Zubia, 2017). However, these education tools are limited in scope and are only suitable for specific circuits within a subject area. To improve applicability to a broader array of circuit types, an educational system for supporting virtual and physical circuit-construction was developed (Takemura, 2018). These conventional systems allow a student to construct a circuit using a breadboard (Figure 1(a)). Here, soldering of the circuit components (e.g., devices and cables) is not performed and the components' terminals are only plugged into the holes on the upper surface of the breadboard. This breadboard-based construction is suitable for beginners' education because of the safety and reusability. However, the solder-less circuit on a breadboard is inferior in the stability and practical use and therefore, is applicable only to trial construction or construction of small-scale circuits for elementary education. To overcome the shortcoming of the conventional systems, a novel education system for learning electronic circuit construction involving soldering on a universal circuit-board (Figure 1(b)) is proposed. In addition, a web-based instruction set for wiring and soldering on the lower surface of the circuit-board based on a circuit image-processing technique is included.

The rest of this paper is organized in the following manner. Section 2 describes the technical features of the proposed system for instruction of circuit construction with soldering on a universal circuit-board. Section 3 describes the experimental methodology to evaluate the proposed system. Section 4 summarizes the results of the experiments at actual university laboratories. This section also describes the quantitative evaluation of the proposed system. Finally, Section 5 provides the conclusions. 
(a)

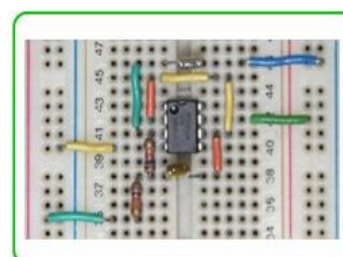

(b)

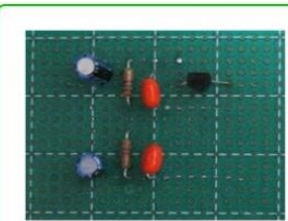

Upper surface

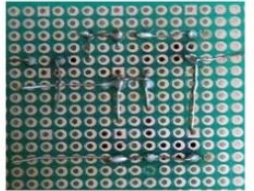

Lower surface

Figure 1. Examples of constructed circuits on the two types of circuit boards: (a) constructed circuit on a solder-less breadboard, and (b) constructed circuit soldered on a universal circuit-board

\section{TECHNICAL FEATURES OF THE SYSTEM}

Figure 2 illustrates the technical features of the proposed system for learning circuit construction involving soldering on a universal circuit-board. This system consists of the individual students' mobile PC and the remote analysis system. A student uses his mobile PC to construct a virtual circuit by handwriting using a graphic editor (Takemura, 2018). Based on the layout of the circuit components on the virtual circuit, the student can efficiently construct a physical circuit by placing circuit devices on the upper surface of the circuit-board and wiring and soldering on the lower surface. The remote analysis system can automatically recognize the structures of the virtual and physical circuits by image processing (e.g., segmentation and pattern recognition) of the constructed circuit images (Figure $2 \mathrm{~B}(3)$ ). In this study, based on the circuit structures obtained from the image processing, an image analysis-based process was developed for automating instructions on demand for wiring and soldering on the lower surface of the circuit-board (Figure 2B(4)). In addition, the developed system enables individual students to simulate their constructed circuits' behaviors (Figure $2 \mathrm{~B}(6)$ and $\mathrm{B}(7)$ ). This circuit simulation is obtained from the translation of the recognized circuit structure into a general circuit description language (simulation program with integrated circuit emphasis (SPICE)) (Rabaey, 2012). Moreover, differences of the SPICE information between the correct circuit and the constructed circuit can identify errors in the student's circuit.

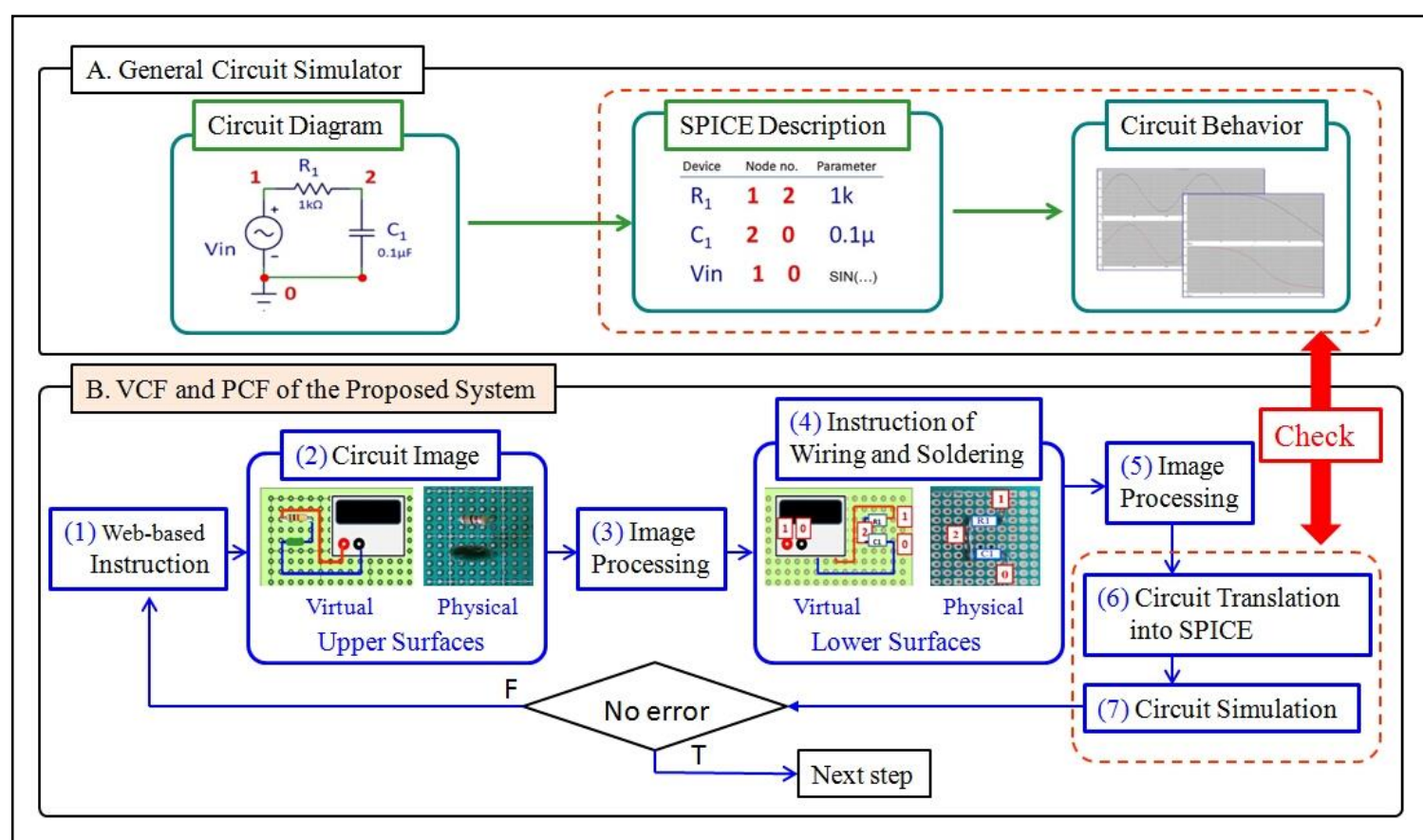

Figure 2. Schematic of the proposed workflow for circuit construction 


\subsection{Web-based Instructions for Circuit Construction}

The proposed education system provides individual students with circuit diagrams and specifications of the circuit to be constructed available through common web-based databases. Based on the information obtained from the circuit translation, the proposed system sends messages to individual students during circuit construction and instructs them to inspect and correct their results in the following manner:

1) When incorrect components or faulty wiring and soldering are detected from a circuit image, the analysis system indicates the errors and instructs the student to check and correct them.

2) When the analysis system detects a serious error (e.g., short circuit), the system sends the student a critical warning to correct the incorrect section.

3) The analysis system requests the student to check whether the simulated behavior of the constructed circuit corresponds to the circuit's specification.

\subsection{Virtual Circuit Construction Function}

Figure 2B also shows the virtual circuit construction function (VCF) of the proposed system. The VCF aids in designing the layout of circuit devices on the upper surface of the universal circuit-board and provides instructions on wiring the circuit devices and the soldering positions. Using a graphic editor, a student can design the layout of the circuit components as an image of the upper surface of a circuit-board by placing virtual circuit components downloaded from a database. As shown in Figure 2B(2), the student draws colored lines to indicate the wiring of each device. Based on the recognition of the virtually constructed circuit, the proposed system can automatically provide instructions for wiring and soldering on the lower surface of the circuit-board (Figure 2B(3) and (4)). This function also simulates the circuit operation and individual students can observe the circuit behavior on their tablet-type PCs. The presence and location of incorrect components in the virtual circuit are determined by analyzing the difference of the SPICE information between the correct circuit and the virtual circuit. When faulty parts or incorrect behavior of the virtual circuit is indicated, the system provides the student with instructions or warnings as described in Section 2.1. Conversely, when correct circuit behavior and components are indicated, the system instructs the student to construct a physical circuit using the function described below.

\subsection{Physical Circuit Construction Function}

Figure 2B also illustrates the physical circuit construction function (PCF) of the proposed system. In accordance with the layout of the virtual circuit preliminary constructed using VCF, a student constructs the physical circuit and transmit an image to the remote analysis system (Figure $2 \mathrm{~B}(2)$ ). This function automatically performs image processing to recognize the circuit's structure and translates the structure into SPICE. Based on the recognized circuit information, the PCF can instruct the wiring and soldering on the image of the lower surface of the circuit-board (Figure $2 \mathrm{~B}(3)$ and $2 \mathrm{~B}(4)$ ). This function also enables the simulation of the circuit operation, and individual students can observe the circuit behavior on their tablet-type PCs. To detect faulty parts in the physical circuit and simulate the circuit behavior, the PCF performs the same processes as the VCF. The presence and location of incorrect components in the circuit are determined by analyzing the difference of the SPICE information between the correct circuit and the physically constructed circuit. When the system indicates faulty parts or incorrect behavior of the physical circuit, the system provides the student the web-based instruction for correcting them. If no faults are identified, the system indicates that the student can proceed with further experiments using the physical circuit.

\section{METHODOLOGY FOR EVALUATING THE PROPOSED SYSTEM}

The effectiveness of the proposed combination of virtual and physical systems was evaluated by the comparison of two groups of students aged 19-21 at Tokyo University of Agriculture and Technology. 
Group-1 was comprised of 20 students instructed to construct an audio amplifier circuit, as shown in Figure 3(a), by conventional methods. Group-2 consisted of 20 students who performed the same circuit construction using the new system. Groups-1 and -2 were evaluated in 2018 and 2019, respectively. The system for the circuit construction used by the students in Group-2 is shown in Figure 3(b). Prior to the experiments, questionnaires were provided to ascertain the degrees of individual students' experience in circuit construction with soldering. Based on this, each student was subdivided into three experience categories as listed below:

A: Experienced in circuit construction with soldering in addition to practice exercises at school

B: No experience except for practice exercises at school

C: No experience

The number of students belonging to each experience category is shown in Figure 3(c) and indicates that the majority of students belonged to category B. All students had learned construction of elementary circuits with soldering as part of compulsory education in junior high schools in Japan and therefore there were no students belonging to category $\mathrm{C}$.

The students in Group-1 were tasked to construct circuits based on the conventional method listed below within a time limit of $3.5 \mathrm{~h}$ :

(G1-1) Lecture on circuit construction and soldering by an instructor ( $0.5 \mathrm{~h})$

(G1-2) Physical circuit construction using the necessary circuit components provided by the instructor

(G1-3) The instructor inspects the constructed circuit. If an incorrect part is discovered, this student corrects it under the instructor's guidance ( $2 \mathrm{~h}$ for (G1-2) and (G1-3))

(G1-4) Measurement of the characteristics of the constructed circuit using experimental equipment $(1 \mathrm{~h})$

The individual students in Group-2 were tasked to construct circuits using the proposed new system within $3.5 \mathrm{~h}$ according to the following processes:

(G2-1) Lecture on circuit construction and soldering by an instructor $(0.5 \mathrm{~h})$

(G2-2) Construction of the circuit using VCF and PCF

(G2-3) The instructor inspects the physical circuit. If an incorrect part is discovered, this student corrects it under the instructor's guidance ( $2 \mathrm{~h}$ for $(\mathrm{G} 2-2)$ and $(\mathrm{G} 2-3))$

(G2-4) Measurement of the characteristics of the constructed circuit using experimental equipments (1 h)

(a)

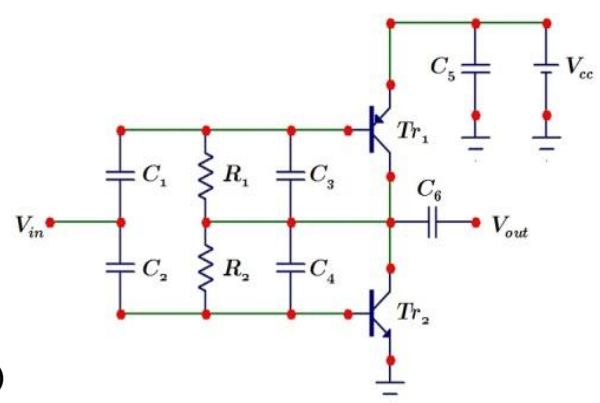

(b)

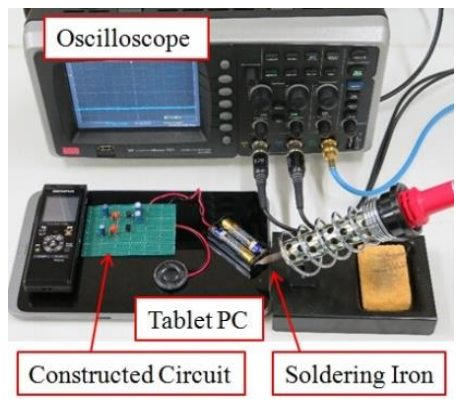

(c)

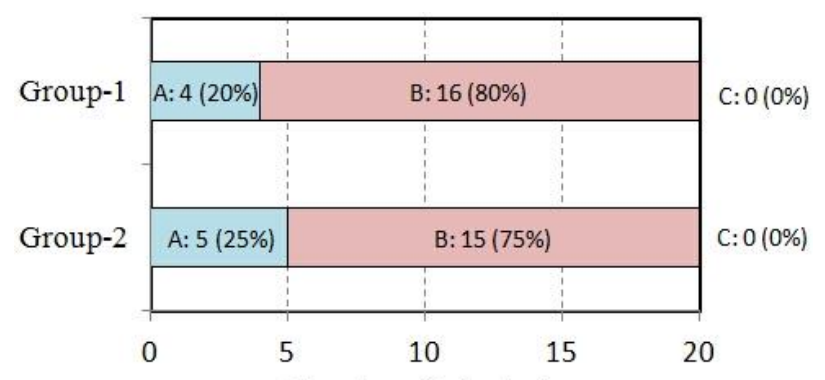

Number of students

Figure 3. Method for evaluation of the proposed system; (a) circuit diagram of an audio amplifier to be constructed in this experiment, (b) system used for circuit construction with Group-2, and (c) distribution of experience categories in circuit construction with soldering for each group 


\section{RESULTS AND DISCUSSION}

Comparison of testing results between Group-1 and Group-2 were evaluated to show the impact of the proposed system on the experiment described in Section 3. Figure 4 shows examples of the constructed circuits and simulation results obtained from a student in Group-2. Figure 4(a) shows the images of the constructed circuit corresponding to the diagram shown in Figure 3(a). Based on the layout of the virtually constructed circuit using VCF (Figure 4(v1)), the physical circuit (Figure 4(p1)) was constructed using PCF. Figure 4(b) shows the results of automated circuit recognition of the circuit images and the web-based instruction for wiring and soldering on the lower surface of the circuit-board. Figure 4(c) shows the results of the virtual measurement; the circuit translation into SPICE was obtained from the circuit recognition, and the translated SPICE enables the student to simulate the circuit's behavior.

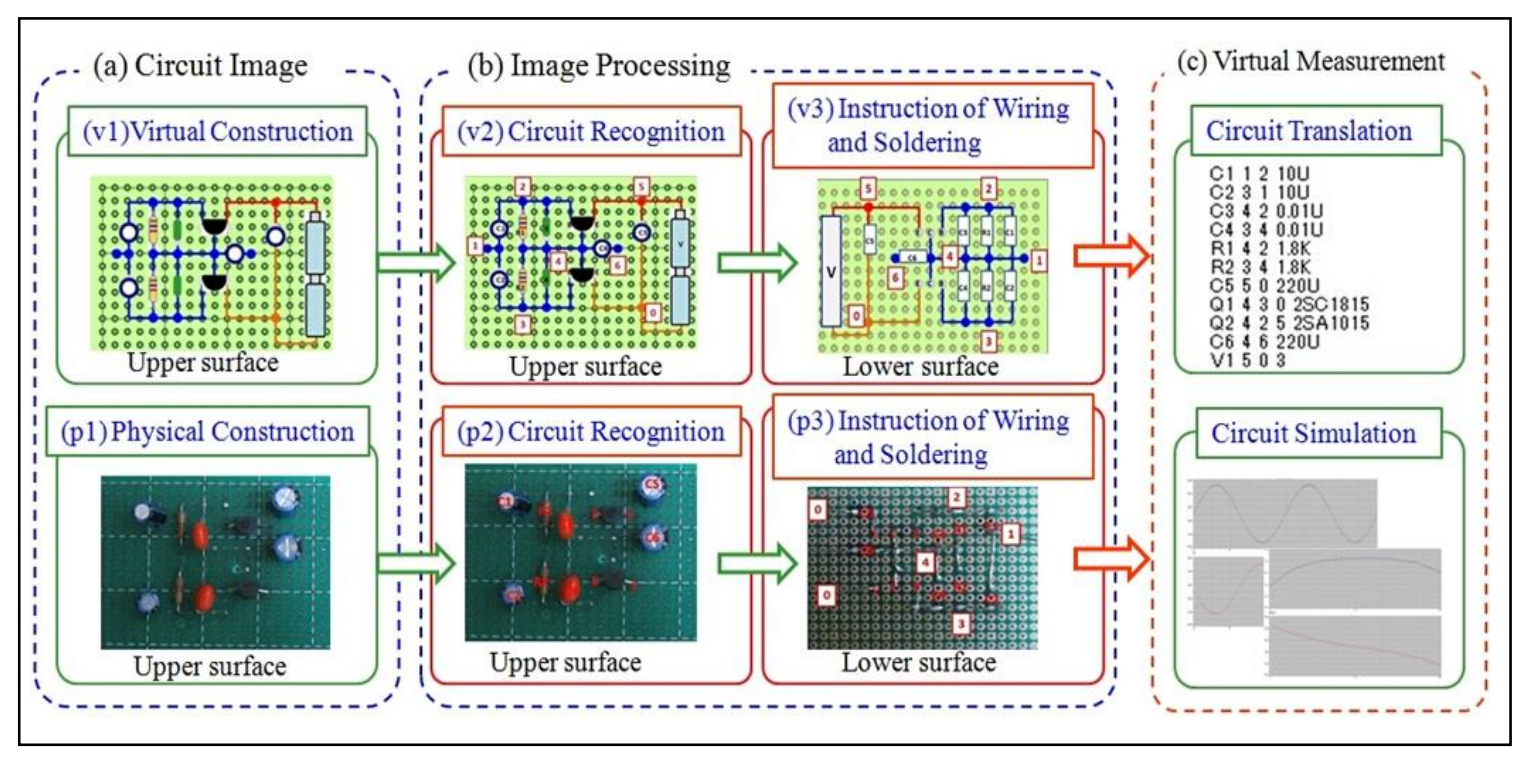

Figure 4. Results of the construction and simulation of the virtual and physical circuits constructed by a student in Group-2 using VCF and PCF of the proposed system

Figure 5(a) shows that the averages and ranges of the required time for the circuit construction of each experience category in Group-2 were shorter than that of Group-1. The average required time of the students in category B in Group-2 was close to that of category A in Group-1. These results indicate that the use of the proposed system decreased the time for circuit construction with soldering regardless of the students' experience. Figure 5(b) indicates the occurrence of errors in the constructed circuits that were discovered by the instructors during the process. As shown in this figure, ten (50\%) students in Group-1 had errors in circuit construction that were observed by the instructor. Conversely, no errors in the circuits constructed by the students in Group-2 were noted. These results indicate the effectiveness of the proposed system for circuit construction with soldering on a universal circuit-board.

Through the evaluation process, positive responses were obtained from the instructors, which provide further validation of the effectiveness of the proposed system. These comments show that:

- The proposed system can improve the accuracy and efficiency of circuit construction with soldering.

- Combination of VCF and PCF is effective for avoiding serious errors that could cause accidents (e.g., a short circuit or inappropriate power supply) when operating the physical circuits.

- The web-based instructions given during VCF and PCF decrease an instructor's load and also increase safety of experiments involving circuit construction with soldering.

From the students in Group-2, the positive responses indicating the usefulness of the proposed system were obtained and include:

- VCF was a useful tool as the image of the virtual circuit layout helped to efficiently construct the physical circuit. 
- The automated instructions based on the circuit image-processing increased the efficiency for wiring and soldering on the lower surface of the circuit-board.

To improve the applicability and usefulness of the system, the following studies should be implemented:

- evaluation test by beginners with no experience in soldering

- application to larger scale circuits

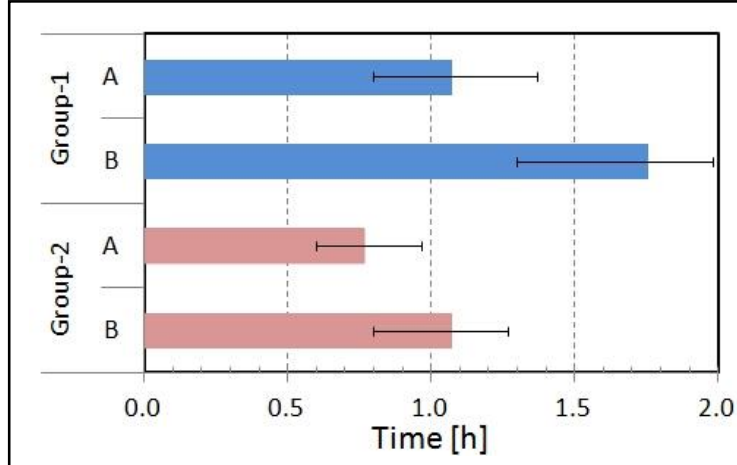

(a)

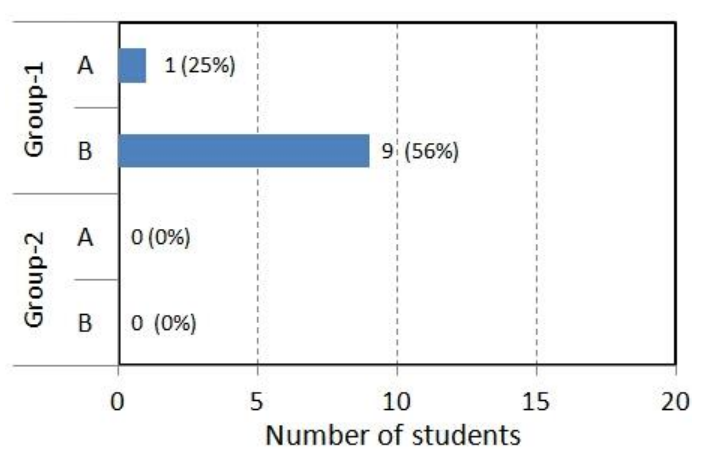

(b)

Figure 5. Results of the comparison test between Group-1 and Group-2; (a) average and range of the required time for the circuit construction of each group and experience degree, (b) the number of students whose circuits included errors

\section{CONCLUSION}

A novel web-based education system for circuit construction involving soldering on a universal circuit-board is demonstrated. The proposed system automatically recognizes the image of constructed circuits and can instruct the student in wiring and soldering on the lower surface of the circuit-board. A comparison study between two groups of university students demonstrated the effectiveness of the proposed system for accurate circuit construction with soldering on a circuit-board. Improvement of the web-based construction and image processing techniques are necessary for the applicability to more complex and larger scale circuits.

\section{ACKNOWLEDGMENT}

This study was partly supported by a Grant-in-Aid for Scientific Research (KAKENHI) 19K03079 from the Japan Society for the Promotion of Science.

\section{REFERENCES}

Garcia-Zubia, J., et al., 2017, Empirical analysis of the use of the VISIR remote lab in teaching analog electronics, In IEEE Trans. Education, Vol. 60, No. 2, pp. 149-156.

Rabaey, J. M., The Spice Page, <URL: http://bwrc.eecs.berkeley.edu/Classes/IcBook/SPICE/> (accessed July 18, 2019).

Reisslein, J., et al., 2013, Circuits kit K-12 outreach: impact of circuit element representation and student gender, In IEEE Trans. Education, Vol. 56, No. 3, pp. 316-321.

Rodriguez-Sanchez, M. C., et al., 2016, An embedded systems course for engineering students using open-source platforms in wireless scenarios, In IEEE Trans. Education, Vol. 59, No. 4, pp. 248-254.

Takemura, A., 2018, e-Learning system for electronic circuit construction using handwriting recognition and mixed reality technique, Proc. IADIS Conference e-Learning 2018, pp. 161-166, Madrid, Spain. 